\#131 Avaliação da perda dentária e presença de reabilitação protética em idosos

Nélio Veiga*, Joana Paiva Alves, Telma Filipa

Nunes Silva Moreira, Rafaela Pereira Guilherme,

Andreia Cristina Silveira Façanha, Mariana de Oliveira Pimenta

Instituto de Ciências da Saúde - Universidade Católica

Portuguesa

Objetivos: Analisar a perda dentária e reabilitação protética numa amostra de idosos institucionalizados em Viseu.

Materiais e métodos: Foi realizado um estudo observacional transversal numa amostra final de 208 idosos institucionalizados. A recolha de dados realizou-se em dois lares de Viseu, onde foi efetuada uma observação intraoral e aplicação de um questionário para avaliação de parâmetros do estudo como: quantidade de peças dentárias em boca, nível sociocultural, tipo de reabilitação oral e presença de satisfação com as próteses dentárias. A análise estatística foi realizada através do programa SPSS versão 23.0 em que as prevalências foram apresentadas sobre a forma de percentagens.

Resultados: Do total da amostra analisada, 77,4\% não realizam uma consulta médico-dentária há mais de 12 meses. No que concerne à avaliação da perda dentária, mais de metade dos idosos apresentava ausência total de dentes. Verificou-se também, que $64,9 \%$ estão reabilitados nas duas arcadas (superior e inferior), apresentando, na sua maioria, próteses totais removíveis. A generalidade dos idosos refere satisfação $(57,7 \%)$ e conforto em relação às próteses. A presença de um maior número de peças dentárias encontra-se associada a um mais elevado nível de escolaridade por parte dos idosos $(p=0,03)$.

Conclusões: A perda dentária e o tipo de reabilitação encontram-se relacionados com o nível sociocultural do idoso, sendo que quanto menor for este fator, menor é a quantidade de peças dentárias. Os pacientes sentem-se, na sua maioria, satisfeitos com a prótese. No estudo, verificou-se uma reduzida procura pelos cuidados médico-dentários. Torna-se essencial a implementação de estratégias de saúde oral para a sensibilização da população idosa.

http://doi.org/10.24873/j.rpemd.2018.11.365

\#132 Timing de escovagem com pasta fluoretada na microdureza de superfície de esmalte erodido

Inês Pádua*, Carla Ascenso, Joana Leite do Carmo, André Peixoto, Ana Cristina Garcia Matos Manso

Instituto Universitário Egas Moniz;CiiEM - Centro de Investigação Interdisciplinar Egas Moniz, Caparica

Objetivos: Pretende-se avaliar o timing da escovagem com uma pasta dentífrica fluoretada na reposição dos valores de microdureza de superfície do esmalte erodido artificialmente com uma bebida energética.

Materiais e métodos: A amostra foi obtida a partir de 18 molares humanos (intactos, livres de cárie e fraturas). Cada dente foi seccionado em 4 fragmentos ( $4 \times 4 \mathrm{~mm}$ ) originando 70 espécimenes de esmalte, divididos aleatoriamente em 7 grupos distintos ( $\mathrm{n}=10)$ : $\mathrm{G} 1$, grupo controlo, nenhum procedimento aplicado; G2, grupo controlo, desafio erosivo com RedBull ${ }^{\circledR} 3$ minutos, 4 vezes por dia durante 5 dias e espécimenes mantidos em água bidestilada; G3, grupo controlo, desafio erosivo idêntico a G2, mas os espécimenes foram mantidos em saliva artificial; G4, desafio erosivo idêntico a G3, seguido (0 min) de escovagem com pasta dentífrica fluoretada (Oral $\mathrm{B}^{\circledR}$ pro-expert); G5, idêntico a G4, mas escovagem realizada 30 min após o desafio erosivo; G6, idêntico a G4, mas escovagem realizada 120 min após o desafio erosivo; G7, idêntico a G4, mas escovagem realizada 240 min após o desafio erosivo. A microdureza de Vickers foi medida no aparelho HSV-30T (Shimadzu Corporation). Os dados recolhidos foram analisados estatisticamente por aplicação de um teste ANOVA a um factor (intervalo de confiança de 95\%), seguido de comparação múltipla pelo teste de contrastes (software SPSS, versão 24).

Resultados: Obtiveram-se os seguintes valores médios

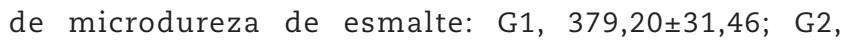
$314,02 \pm 49,60 ; G 3,321,80 \pm 27,67 ; G 4,275,36 \pm 50,10 ; G 5$,

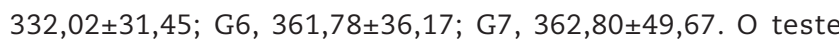
ANOVA a um factor, $F(6,63)=7,441$ revelou existirem diferenças significativas entre os grupos de estudo $(p=0,001)$. 0 teste de contrastes revelou igualdade estatística entre os grupos G1, G6 e G7 ( $p=0,285$ ), entre os grupos G2, G3 e G5 $(p=0,372)$, e diferenças significativas de todos os grupos com o grupo G4 $(\mathrm{p}=0,001)$

Conclusões: Os resultados sugerem o melhor timing da escovagem dos dentes como sendo os $120 \mathrm{~min}$ após o ataque erosivo do esmalte.

http://doi.org/10.24873/j.rpemd.2018.11.366

\#133 Avaliação da saúde oral das crianças institucionalizadas em Viseu

Liliana Sena Silva*, Catarina Fernandes Oliveira, Letícia Ferreira, Mariana Santiago, Andreia Figueiredo, Mariana Seabra

Universidade Católica de Viseu, Universidade Católica Portuguesa de Viseu

Objetivos: Avaliar o estado de saúde oral das crianças institucionalizadas em Viseu e as reais necessidades de tratamentos dentários.

Materiais e métodos: Estudo observacional transversal aplicado a todas as crianças institucionalizadas em Viseu. Foi preenchida uma ficha clínica adaptada da Associação Americana de Odontopediatra e efetuou-se observação intraoral. Foi preenchido o Índice CPOD e realizada a pesagem e a medição, foi ainda realizado às crianças, com indicação clinica para tal, uma ortopantomografia.

Resultados: Das 100 crianças 55 eram do género masculino e 45 do género feminino. Idade média de 12,21 /- 4,41 anos, entre os 1 e 18 anos sendo que $70 \%$ estavam com peso saudável e $81 \%$ das crianças já tinha ido previamente ao Médico Dentista, valores esses que se encontram de acordo com 
os dados obtidos a nível nacional (PNPSO). A média do índice CPOD foi de 3,43/- 3,58, superior à descrita pela OMS para Portugal $(1,2$ a 2,6$)$ estando, a nossa amostra, classificada como risco de cárie moderado. Quanto maior a idade maior o valor do índice $\operatorname{CPOD}(\mathrm{p}=0,000<0,05)$ o que está relacionado com o facto dos dentes se encontrarem erupcionados há mais tempo o que dá mais tempo para que haja deterioração dos mesmos, também as crianças que já tinham ido previamente ao Médico Dentista apresentavam um valor de CPOD significativamente maior ( $p=0,000<0,05)$, esta é uma associação positiva pois indica que as crianças que de facto tinham uma pior saúde oral estavam a ser seguidas no médico dentista previamente.

Conclusões: Percebemos assim a necessidade de medidas de prevenção primária e estratégias de promoção de saúde oral para as crianças que residem em Instituições tuteladas pelo Estado, bem como para as pessoas que as auxiliam no seu dia-a-dia.

http://doi.org/10.24873/j.rpemd.2018.11.367

\#134 Atitudes e comportamentos de saúde oral em estudantes universitários

Joana Leonor Pereira*, Mariana Mota, Bárbara Cunha, Ana Messias, Teresa Xavier, Ana Luisa Costa

Área de Medicina Dentária, Faculdade de Medicina, Universidade de Coimbra

Objetivos: As atitudes e comportamentos de higiene oral e a regularidade da monitorização da condição oral por parte do profissional de saúde variam entre indivíduos, sendo influenciados por diferentes fatores descritos na literatura, como crenças individuais, personalidade e estilo de vida, percepção cultural, género, área de residência, nível e tipo de escolaridade do próprio e progenitores, entre outros. Este trabalho teve como objetivo caraterizar as atitudes e comportamentos de saúde oral (SO) de uma amostra de alunos de um Mestrado Integrado em Medicina Dentária de uma universidade portuguesa tendo por base estudos semelhantes em populações universitárias distintas, avaliando igualmente a potencial influência do seu percurso académico na adopção e futuro aconselhamento de atitudes preventivas.

Materiais e métodos: Para a recolha de dados foi elaborado um questionário baseado em publicações disponíveis, confidencial e autoaplicável, incluindo dados gerais, situação no ensino superior, nível de instrução dos pais, percepção, comportamentos e atitudes de SO (instrumento HU-DBI Hiroshima University-Dental Behavioral Inventory) e hábitos de higiene oral. Este questionário foi aplicado aos alunos do primeiro e último anos que se mostraram disponíveis para o seu preenchimento, tendo os dados sido estatisticamente tratados com recurso a análises descritiva e inferencial (Qui-quadrado, Mann-Whitney e correlação não paramétrica de Spearman).

Resultados: Foram incluídos na amostra 64 indivíduos, de ambos os sexos, com uma média de idades de 21,28 anos $( \pm 3,56)$. No respeitante ao HU-DBI os scores médios registados neste estudo foram de 7,74 e 8,43 no $1 .^{\circ}$ e $5 .^{\circ}$ anos, respetivamente, com uma discrepância marginalmente não-significativa. Apenas se constaram diferenças estatisticamente mais marcadas no referente ao uso do fio dentário e consumo de alimentos açucarados.

Conclusões: Dentro das limitações do presente estudo verificou-se que o percurso académico não parece influenciar o nível de conhecimentos e os hábitos de SO dos alunos integrantes desta amostra, sublinhando-se que os alunos do $1 .{ }^{\circ}$ ano manifestaram, algo surpreendentemente, conhecimentos, atitudes e comportamentos de SO superiores aos reportados por outros autores, ainda que semelhantes aos seus congéneres finalistas.

http://doi.org/10.24873/j.rpemd.2018.11.368

\section{\#135 Clínica e tratamento do Líquen Plano Oral da gengiva. Estudo retrospetivo}

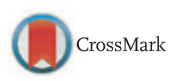

Inês Henriques*, Ana Catarina Pinto, Inês Lourenço Cardoso, Rita Montenegro, Helena Rebelo, António Mano Azul

Clínica Integrada de Medicina Oral - Departamento de Periodontologia, Clínica Integrada de Medicina Oral Departamento de Cirurgia e Medicina Oral

Objetivos: Avaliar e descrever as características clínicas do Líquen Plano Oral (LPO) eritematoso / erosivo / ulcerativo das gengivas bem como as opções e resultados do tratamento dos doentes em estudo.

Materiais e métodos: Estudo retrospetivo pela análise de 9595 fichas clinicas de doentes observados numa clinica dentária em Lisboa, entre 2005 e 2016. Foi efetuada uma estatística descritiva, inferencial (Teste do Chi-quadrado, com significância de 5\%).

Resultados: Dos 263 doentes com diagnóstico de LPO (correspondente a $2,7 \%$ da população geral dos doentes do consultório), 91 (34,6\%) apresentaram lesões gengivais de líquen do tipo 'gengivite descamativa' (LPO eritematoso / erosivo / ulcerativo gengival). $84,6 \%$ destes doentes eram mulheres e a idade média foi de 64 anos. $46,2 \%$ dos doentes apresentavam lesões em ambos os maxilares e $71,4 \%$ tinham lesões bilaterais. 91\% dos doentes tinham também lesões de LPO noutras localizações, ou seja, em 9\% da população estudada as lesões gengivais eram a única manifestação da doença. $67 \%(n=56)$ dos doentes apresentaram sintomas (desconforto / dor ligeira / dor grave ou problemas estéticos) pelo que iniciaram terapêutica imunossupressora tópica (Grupo T $=29$ ) ou terapêutica tópica associada à sistémica (Grupo T e $S=27$ ). Na primeira consulta de reavaliação ( $n=48$; Grupo T: 24; Grupo T e S: 24), por volta das 4 semanas, $83 \%$ dos doentes estavam assintomáticos ou tinham melhorado (Grupo T: 79\%; Grupo T e S: $88 \%$ ); 54\% dos doentes do Grupo $\mathrm{T}$ interromperam tratamento iniciando um esquema periódico de reavaliações.

Conclusões: Após um período médio de 2 meses (min: 2 semanas - máx: 20 meses) todos os doentes estavam assintomáticos e sem necessidade de medicação imunossupressora ( $n=45$, com 3 exclusões), sem diferenças estatisticamente significativas $(\mathrm{p}<0,05)$ entre o tratamento tópico e o 